\title{
Gastrin-releasing peptide promotes the migration of vascular smooth muscle cells through upregulation of matrix metalloproteinase- 2 and -9
}

\author{
Hyun-Joo Park ${ }^{1, \#}$, Mi-Kyoung Kim ${ }^{1, \#}$, Yeon Kim ${ }^{1}$, Sun Sik Bae ${ }^{3}$, Hyung Joon Kim ${ }^{1}$, Soo-Kyung Bae ${ }^{2}$ E \\ Moon-Kyoung Bae ${ }^{1, *}$ \\ ${ }^{1}$ Department of Oral Physiology, ${ }^{2}$ Department of Dental Pharmacology, BK21 PLUS Project, School of Dentistry, ${ }^{3}$ Department of \\ Pharmacology, Pusan National University School of Medicine, Pusan National University, Yangsan 50610, Korea
}

\begin{abstract}
Gastrin-releasing peptide (GRP) has been reported to be implicated in the pathogenesis of inflammatory disorders. The migration and proliferation of vascular smooth muscle cells (VSMCs) are key components of vascular inflammation that leads to the development of atherosclerosis. The present study aimed to investigate the molecular effect of GRP on VSMC proliferation and migration. We report that GRP significantly enhanced the proliferation and migration of rat VSMCs. GRP increased mRNA and protein expression of matrix metalloproteinase-2 and -9 (MMP-2/9) in VSMCs. The induction of MMP-2/9 by GRP was regulated by the activation of the signal transducer and activator of transcription-3 (STAT3). In addition, STAT3-knockdown of VSMCs by siRNA or blockade of the GRP receptor inhibited GRP-induced migration of VSMCs. Taken together, our findings indicate that GRP promotes the migration of VSMCs through upregulation of MMP-2/9 via STAT3 activation. [BMB Reports 2017; 50(12): 628-633]
\end{abstract}

\section{INTRODUCTION}

Gastrin-releasing peptide (GRP), a primary member of mammalian bombesin-like peptides, is a small regulatory peptide that has been implicated in various cellular responses including cell growth, proliferation, survival, inflammation, and angiogenesis under physiological and pathological situations (1). GRP binds with high affinity to the GRP receptor in an autocrine, paracrine, or neuroendocrine manner to

*Corresponding author. Tel: +82-51-510-8239; Fax: +82-51-5108238; E-mail: mkbae@pusan.ac.kr

${ }^{\#}$ These authors contributed equally to this work.

https://doi.org/10.5483/BMBRep.2017.50.12.158

Received 14 August 2017, Revised 31 August 2017, Accepted 11 October 2017

Keywords: Gastrin-releasing peptide, Matrix metalloproteinase-2/9, Migration, STAT-3, Vascular smooth muscle cells regulate diverse biological functions (2). The GRP receptor is a specific member of the G-protein-coupled receptor family and is found in different types of normal and cancerous tissues (3).

The vascular inflammatory response involves the adherence and recruitment of inflammatory cells (lymphocytes, neutrophils, and monocytes) to vascular cells (endothelial cells and vascular smooth muscle cells (VSMCs) (4). Upon exposure to pro-inflammatory stimuli, vascular endothelial cells and VSMCs regulate the expression of cell adhesion molecules, growth factors, cytokines, and matrix metalloproteinases (MMPs), which can lead to the progression of cardiovascular diseases such as atherosclerosis (5). GRP has been shown to mediate the migration and recruitment of neutrophils, which acts as inflammatory mediators (6). We have previously demonstrated that GRP directly induces endothelial dysfunction through the upregulation of cell adhesion molecules in endothelial cells (7). However, little attention has been devoted to the potential role of GRP on inflammatory responses in VSMCs.

In this study, we examined the effects of GRP on VSMC migration and proliferation, which have been linked to the development and progression of atherosclerosis (8). We also demonstrated that GRP-induced migration of VSMCs is mediated by the upregulation of MMP-2/9 and have explored the underlying mechanism(s).

\section{RESULTS}

GRP induces proliferation and migration in VSMCs

VSMCs were treated with different concentrations of GRP for up to $72 \mathrm{~h}$, and cell proliferation was measured by the MTT assay. As shown in Fig. 1A, the proliferation rate increased in a dose-dependent manner with increasing GRP concentrations at $48 \mathrm{~h}$ and $72 \mathrm{~h}$, respectively, but no such effects were observed at $24 \mathrm{~h}$. The maximal increase in proliferation, a 1.7-fold increase over control, was induced by GRP. BrdU incorporation assay was employed to further test VSMC proliferation. The results showed that treatment of VSMCs with GRP significantly induced in cell proliferation in a dose- and time-dependent manner (Fig. 1B). The migration of VSMCs is known to be a 


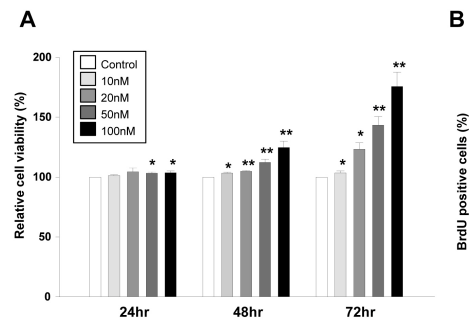

C
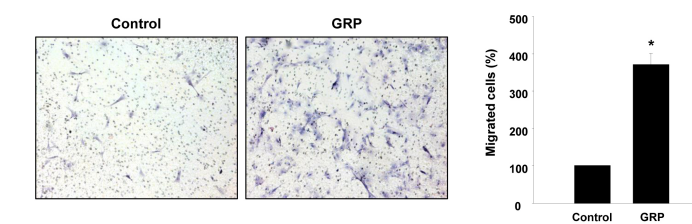

D
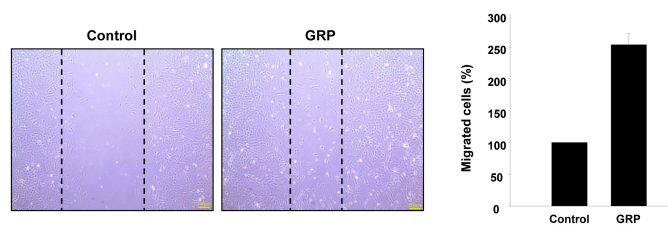

Fig. 1. Effect of GRP on proliferation and migration in vascular smooth muscle cells. (A) VSMCs were exposed to different concentrations of GRP for the indicated times. After treatment, cell proliferation was determined by MTT assay. The data are expressed as percentage \pm SE from at least three different experiments performed in triplicate. $* P<0.05 ; * *<0.01 \mathrm{vs}$. control. (B) VSMCs were incubated with the indicated concentrations of GRP for 48 and $72 \mathrm{~h}$, and the proliferating cells were assayed using BrdU incorporation. ${ }^{*} \mathrm{P}<0.1$ vs. control. (C) VSMCs were seeded on gelatin-coated filters for the migration assay. VSMCs in the absence or presence of GRP (100 nM) were incubated in Transwell chambers for $24 \mathrm{~h}$. The migrated cells were stained with hematoxylin-eosin and photographed. The number of migrated cells was counted. $* P<0.1$ vs. control. (D) The migration capability of VSMCs was analyzed by scratch wound migration assays. The number of cells that migrated beyond the reference line was counted.

key event in the arterial response to injury, vessel development and atherosclerosis (9). In this study, the effect of GRP on VSMC migration was examined using a modified Boyden's chamber assay. We found that GRP significantly enhanced the migration of VSMCs, by approximately 3.8-fold (Fig. 1C). We also performed a scratch wound migration assay to qualitatively observe the effect of GRP-induced motility of VSMCs. As shown in Fig. 1D, GRP treatment increased the number of cells that migrated into the scratch wound compared to that observed with control VSMCs.

\section{GRP promotes the expression and activity of MMP-2 and MMP-9 in VSMCs}

MMP-2 and MMP-9 are known to be major proteinases that contribute to the migration ability of VSMCs (10). Thus, we

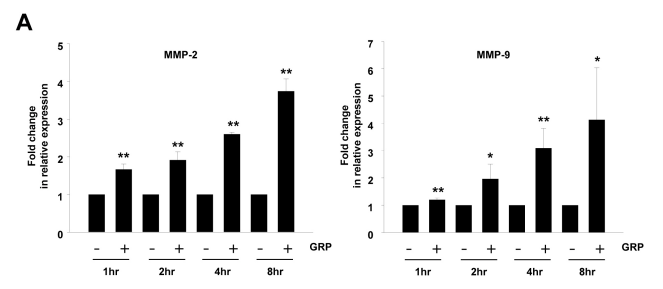

B
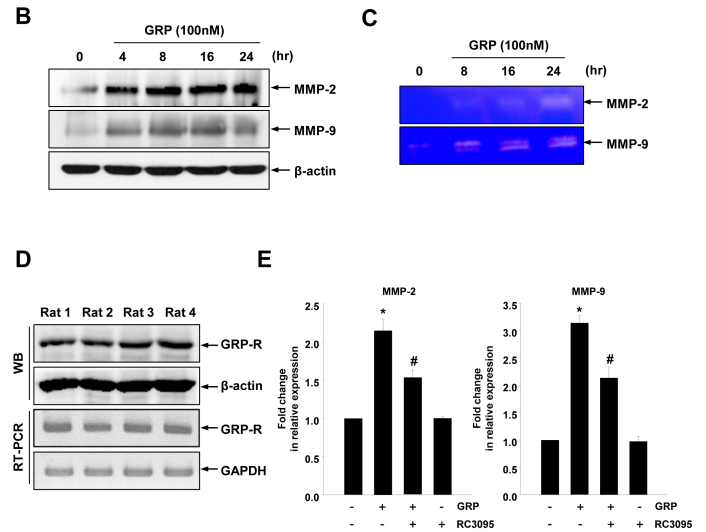

Fig. 2. Effect of GRP on the expression and activation of MMP-2/9 in vascular smooth muscle cells. (A) VSMCs were incubated with GRP $(100 \mathrm{nM})$ for the indicated times. Total RNA was isolated and then analyzed by real-time PCR using primers specific for rat MMP-2 and MMP-9. The expression level of the control (untreated) was set to 1 , and the values are normalized to the $\beta$-actin mRNA levels. ${ }^{* P}<0.05 ; *{ }^{*}<0.01$ vs. control. (B) MMP-2 and MMP-9 protein levels were examined by western blotting using anti-MMP-2 and anti-MMP-9 antibodies. $\beta$-actin served as the loading control. (C) VSMCs were exposed to GRP for the indicated times. The conditioned medium was concentrated and subjected to gelatin zymography. (D) Protein and mRNA expression levels of GRP-R was observed by western blotting and RT-PCR, respectively. $\beta$-actin and GAPDH served as the loading control. (E) VSMCs were incubated with GRP (100 $\mathrm{nM})$ alone or in combination with RC-3095 (100 $\mathrm{nM})$ for $8 \mathrm{~h}$. Using real-time PCR, the expression levels of MMP-2 and MMP-9 mRNA were also quantified. The expression level of the control (untreated) was set to 1 , and the values were normalized to the $\beta$-actin mRNA levels. ${ }^{*} \mathrm{P}<0.05$ vs. control; ${ }^{\#} \mathrm{P}<0.05$ vs. GRP alone.

investigated the role of GRP on the expression of MMP-2 and MMP-9 in VSMCs. Cells were treated with GRP for the indicated times, and levels of MMP-2 and MMP-9 mRNA were measured real-time quantitative PCR analysis. As shown in Fig. 2A, GRP significantly induced the expression of MMP-2 and MMP-9 mRNA in a time-dependent manner. However, GRP had little effect on the mRNA expressions of MMP-1, MMP-3, MMP-7, MMP-12, MMP-13, and MMP-14 (data not shown). The protein levels of MMP-2 and MMP-9 in GRP-treated VSMCs were further analyzed by western blot analysis. Consistent with results of the real-time quantitative PCR analysis, GRP significantly induced the protein levels of MMP-2 and MMP-9 in VSMCs (Fig. 2B). Gelatin zymography showed that GRP 
significantly increased levels of mature and active MMP-2 and MMP-9 proteins (Fig. 2C). Recent studies showed that the effects of GRP are known to be mediated by the GRP receptor in many cell types (11). The presence of GRP-R mRNA and protein in VSMCs was confirmed by RT-PCR and western blot analysis, respectively (Fig. 2D). Next, we tested the effect of pretreatment with RC-3095, a selective GRP-R antagonist (12), on the increase in expression of MMP-2 and MMP-9 mRNA caused by GRP. As expected, GRP induced the expression of MMP-2 and MMP-9 mRNA compared with that in the untreated control, which was suppressed by pretreatment with RC-3095 (Fig. 2E).

\section{GRP-induced MMP-2/9 expression is mediated by JAK2/STAT3 signaling pathways}

JAK2/STAT3 signaling is required for expression of MMP-2 and MMP-9 in VSMCs (13). We determined whether GRP stimulates

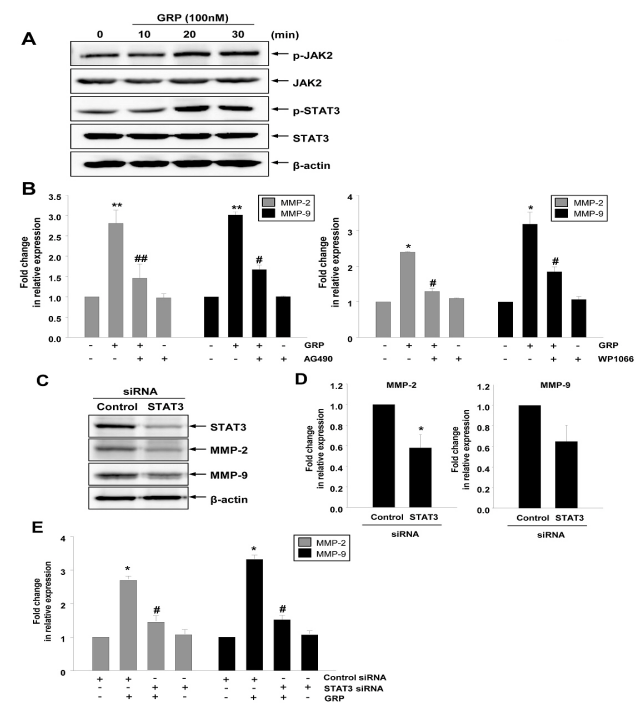

Fig. 3. Effect of JAK2/STAT3 signaling on GRP-induced MMP-2/9 expression. (A) VSMCs were incubated with GRP (100 nM) for the indicated times. Western blots were probed with p-/total-JAK2 and p-/total-STAT3 antibodies. $\beta$-actin served as the loading control. (B and C) VSMCs were pretreated with AG490 (20 $\mu \mathrm{M})$ or WP1066 $(10 \mu \mathrm{M})$ for $30 \mathrm{~min}$ before exposure to GRP (100 $\mathrm{nM})$. (B) After $8 \mathrm{~h}$ of GRP treatment, total RNA was isolated and then analyzed by real-time PCR using primers specific for rat MMP-2 and MMP-9. ${ }^{* P}<0.05 ; * * \mathrm{P}<0.01$ vs. control; ${ }^{\#} \mathrm{P}<$ $0.05 ; \# P<0.01$ vs. GRP alone. (C and D) VSMCs were transiently transfected with control siRNA or STAT3 siRNA for 48 h. (C) Cell extracts were subjected to western blot analysis for detecting STAT3, MMP-2 and MMP-9 protein levels. $\beta$-actin served as the loading control. (D) Total RNA was isolated and then analyzed by real-time PCR using primers specific for MMP-2 and MMP-9. ${ }^{*} P<0.05$ vs. control. (E) Transfected VSMCs with control siRNA or STAT3 siRNA were incubated with GRP (100 $\mathrm{nM}$ ). Total RNA was isolated and then analyzed by real-time PCR using primers specific for rat MMP-2 and MMP-9. ${ }^{* P}<0.05$ vs. control; ${ }^{\#} \mathrm{P}<0.05$ vs. GRP alone. the activation of JAK2 and STAT3 in VSMCs. As shown in Fig. $3 \mathrm{~A}$, treatment of VSMCs with GRP increased the phosphorylation of JAK2 and STAT3 in a time-dependent manner, and the maximal activation was observed after $30 \mathrm{~min}$ of GRP treatment. Next, to elucidate the involvement of JAK2/STAT3 signaling in GRP-induced MMP-2/9 expressions, we performed real-time PCR analysis. As shown in Fig. 3B, pretreatment with the JAK2/STAT3 inhibitors, AG490 or WP1066 (14, 15), significantly decreased the mRNA levels of MMP-2 and MMP-9 otherwise induced by GRP. To confirm that the expression of MMP-2 and MMP-9 was directly regulated by STAT3, we used a STAT3-targeted siRNA or a control siRNA. We demonstrated that the protein and mRNA levels of MMP-2 and MMP-9 were decreased by STAT3-knockdown in VSMCs with siRNA (Fig. 3C and D). In the presence of STAT3-targeted siRNA, the mRNA expression of GRP-induced MMP-2 and MMP-9 was markedly reduced compared with that in control

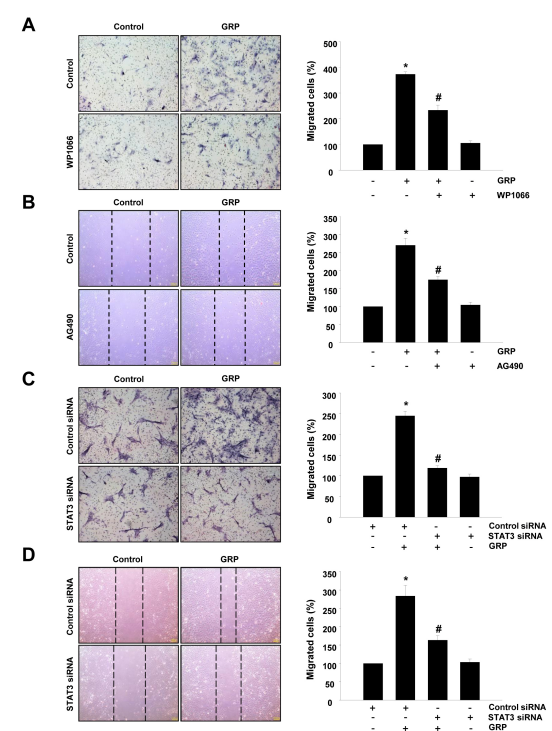

Fig. 4. Effect of JAK2/STAT3 signaling on GRP-induced vascular smooth muscle cell migration. (A) VSMCs were seeded on gelatin-coated filters for the migration assay. VSMCs were treated with GRP $(100 \mathrm{nM})$ in the absence or presence of WP1066 (10 $\mu \mathrm{M})$ in Transwell chambers for $24 \mathrm{~h}$. Migrated cells were stained with hematoxylin-eosin and then counted. (B) Scratch wound migration assays were performed on GRP $(100 \mathrm{nM})$ treated VSMCs in the absence or presence of the AG490 (20 $\mu \mathrm{M})$. The number of cells that migrated beyond the reference line was counted. (C and D) VSMCs were transiently transfected with control siRNA or STAT3 siRNA for $48 \mathrm{~h}$. (C) Transfected VSMCs in the absence or presence of GRP $(100 \mathrm{nM})$ were incubated in Transwell chambers for $24 \mathrm{~h}$. The number of migrated cells was counted. (E) Scratch wound migration assay using STAT3 siRNA-transfected VSMCs was performed and then treated with GRP $(100 \mathrm{nM})$ for $48 \mathrm{~h}$. The number of cells that migrated beyond the reference line was counted. Each column represents the mean value of triplicate experiments in each group. ${ }^{*} \mathrm{P}<$ 0.01 vs. control; ${ }^{\#} \mathrm{P}<0.01$ vs. GRP alone. 
siRNA-transfected VSMCs (Fig. 3E).

\section{Involvement of JAK2/STAT3 signaling pathway in GRP-induced VSMC migration}

To examine whether JAK2/STAT3 signaling is involved in GRP-induced migration in VSMCs, we adopted Boyden's chamber migration assay. As shown in Fig. 4A, pretreatment with WP1066 significantly decreased GRP-induced migration of VSMCs. We also performed a scratch wound migration assay to qualitatively observe the effect of AG490 on GRPinduced motility of VSMCs. As expected, AG490 treatment decreased the number of cells that migrated into the scratch wound compared to that observed in GRP-treated VSMCs (Fig. $4 \mathrm{~B})$. Next, to elucidate the role of STAT3 in migration of VSMCs, we knocked down endogenous STAT3 in VSMCs and assessed the rate of GRP-induced migration. As shown in Fig. 4C and D, depletion of STAT3 markedly reduced the ability of GRP to induce VSMC migration.

\section{DISCUSSION}

The proliferation, migration, and accumulation of VSMCs are critical steps in the pathogenesis of intimal hyperplasia after arterial injury and atherosclerosis (16). During the atherosclerotic process, several mediators, such as growth factors and pro-inflammatory cytokines, induce the phenotypic switch of VSMCs from the quiescent, contractile state to the active, synthetic phenotype as well as VSMC proliferation and migration from the media to the subendothelial region $(17,18)$. Activation of STAT3 by JAK2 regulates a variety of biological responses, including proliferation, survival, differentiation, and inflammation (19). It has been reported that STAT3 regulates the phenotypic change of VSMCs by association with myocardin (20). The activation of STAT3 by JAK2 in VSMCs is induced in response to various growth factors, cytokines and hormones, such as platelet-derived growth factor (PDGF)-BB, angiotensin II, vascular endothelial growth factor (VEGF) and interleukin-6 (21). In this study, we showed that GRP markedly stimulates the activation of STAT3 in VSMCs. Further investigations will be needed to examine whether GRP could reduce the contractile phenotype or/and induce the synthetic phenotype of VSMCs through the JAK-STAT3 signaling pathway.

The major function of MMPs, which form a family of proteolytic enzymes, is to degrade various components of the extracellular matrix $(\mathrm{ECM})$ and essential in physiological and pathological processes including wound healing, angiogenesis, tumorigenesis, and inflammation (10). Among the MMPs, the gelatinases MMP-2 and -9 have been found to be predominantly upregulated in VSMCs within atherosclerotic plaques and of the mouse atherosclerosis model $(22,23)$. Many studies have identified increased MMP-2 and MMP-9 expression has been coincided with VSMC migration from the media to the intima (10). MMP-9 overexpression increases the migration of VSMCs and alters vascular remodeling in the injured carotid artery
(24). MMP-2 contributes to VSMC migration by the degradation of ECM and non ECM, or by inducing a phenotype switch (25). We demonstrated in this report that GRP upregulates the expression of MMP-2/9 in VSMCs and in turn mediates the migration of VSMCs, which suggesting a pathogenic role of GRP as a MMP-2/9 regulator in the progression of atherosclerosis.

The promoter region of MMPs contains binding sites for nuclear factor- $\kappa \mathrm{B}(\mathrm{NF}-\kappa \mathrm{B})$, Activator Protein-1 (AP-1), PEA3, SP-1, and STAT-3 (26). It has been reported that salusin$\beta$-induced MMP-9 expression is mediated by NF- $\mathrm{BB}$ in VSMCs (27). We previously found that GRP induces NF-kB activation in vascular endothelial cells (7). We also observed that the treatment of GRP slightly stimulated I $\mathrm{kB} \alpha$ phosphorylation and degradation in VSMCs (data not shown). The activation of NF- $\kappa B$ has been reported to be positively or negatively associated with STAT3 activation in different types of human cancers (28). We are currently investigating whether NF- $\mathrm{KB}$ activation or the association of NF- $\kappa B$ and STAT3 contribute to GRP-induced MMP expression and VSMC migration.

In conclusion, our research shows for the first time that GRP increases the migration of VSMCs by promoting STAT3-mediated expression of MMP-2/9. Additional in vivo studies will be required to consider whether intervention targeting the GRP-STAT3 axis may be an efficient strategy for the prevention or treatment of vascular diseases.

\section{MATERIALS AND METHODS}

\section{Reagents and antibodies}

GRP and RC-3095 were purchased from Sigma. AG490 and WP1066 were supplied by Calbiochem and Sigma, respectively. The antibodies for GRP-R and MMP-9, were supplied by Santa Cruz Biotechnology. The antibodies for phospho-JAK2, JAK2, phospho-STAT3, and STAT3 were obtained from Cell Signaling. MMP-2 and $\beta$-actin antibodies were purchased from Abcam and Bioworld Technology, respectively. Horseradish peroxidase-conjugated goat anti-rabbit and anti-mouse IgG were obtained from Thermo Fisher Scientific.

\section{Cell isolation and cell culture}

To isolate VSMCs, Sprague-Dawley rats (3 weeks old) were euthanized via intraperitoneal injection of sodium pentobarbital $(60 \mathrm{mg} / \mathrm{kg}$ ) by a tissue explanting method. Thoracic aorta was isolated and surrounding fat and connective tissues were discarded. Vessels were cut longitudinally and the lumen side was scraped with a razor blade to remove the intima. Vessels were fragmented into 3-5 mm lengths and explanted lumen side down on collagen-coated culture dishes. After seven days of explanting, tissue fragments were discarded and sprouted VSMCs were collected (referred to as P0). The VSMCs were grown in DMEM (Gibco) with 10\% FBS (Gibco) and 1\% antibiotics, at $37^{\circ} \mathrm{C}$ in a humidified $95 \%$ air $/ 5 \% \mathrm{CO}_{2}$ environment. 


\section{MTT assay}

VSMCs $\left(5 \times 10^{4}\right.$ cells $)$ were seeded into 24 -well plates and incubated for 24,48 , and $72 \mathrm{~h}$. After exposure to different doses of GRP, cells were treated with MTT solution (0.5 $\mathrm{mg} / \mathrm{mL}$ ) and analyzed by MTT assay as described (29).

\section{BrdU incorporation assay}

To evaluate cell proliferation, we used an FITC BrdU Flow kit purchased from BD Pharmingen according to the manufacturer's instructions. VSMCs were treated with GRP for 48 and $72 \mathrm{~h}$. VSMCs were then labeled with BrdU for $3 \mathrm{~h}$, washed, fixed and permeabilized with BD Cytofix/Cytoperm buffer. After repeated incubation on ice, washing, and centrifugation, cells were treated with DNase to expose BrdU epitopes for $1 \mathrm{~h}$ at $37^{\circ} \mathrm{C}$, washed, then stained with fluorescent anti-BrdU for 20 $\mathrm{min}$ at room temperature, washed again, and analyzed by FACS Calibur (BD Bioscience).

\section{Boyden chamber migration assay}

Transwells with $8 \mu \mathrm{m}$ porosity polycarbonate membrane inserts were coated with $10 \mu \mathrm{g}$ gelatin. VSMCs were suspended in DMEM as $1 \times 10^{5}$ cells/100 $\mu \mathrm{l}$, and were added to the upper chamber. GRP (100 nM) or WP1066 $(10 \mu \mathrm{M})$ in DMEM was added into the lower chamber. VSMCs migrating through the filter and appearing on the lower side were fixed by careful immersion of the filter into methanol for 1 minute, stained with H\&E solution and cells counted in three random fields per well (30). Each experiment was performed in duplicate and three separate experiments were performed for each group.

\section{Scratch wound migration assay}

VSMCs were seeded in 6-well plates at a concentration of $1 \times$ $10^{6}$ cells/well in $1 \mathrm{ml}$ of serum-free DMEM for $6 \mathrm{~h}$, until an adherent monolayer was obtained. A $10 \mu$ pipette tip was used to create a scratch in the monolayer and the cells were washed 3 times with serum-free medium. The cells were then placed in fresh serum-free medium and treated with either GRP or AG490. Samples were taken at the beginning and after $24 \mathrm{~h}$ of culture in $5 \% \mathrm{CO}_{2}$ at $37^{\circ} \mathrm{C}$. Images of the scratch wounds were taken and measured by Image-J software to calculate the mean and standard deviation. Each experimental group was compared with its respective control. The experiments were repeated three times.

\section{Real-time RT-PCR analysis}

Real-time PCR quantification was performed using a SYBR ${ }^{\circledR}$ Green method (Light Cycler; Roche Applied Science). Cycling parameters included 1 cycle at $95^{\circ} \mathrm{C}$ for $10 \mathrm{~min}$, followed by amplification for 30 cycles at $95^{\circ} \mathrm{C}$ for $10 \mathrm{~s}, 57^{\circ} \mathrm{C}$ for $5 \mathrm{~s}$, and $72^{\circ} \mathrm{C}$ for $7 \mathrm{~s}$. The entire cycling process, including data analysis, took less than $1 \mathrm{~h}$ and was monitored using Light Cycler software (version 4.0). The oligonucleotide primers for realtime PCR were as follows: MMP-2, 5'-ACCGTCGCCCATCAT
CAA-3' and 5'-TTGCACTGCCAACTCTTTTTCT-3'; MMP-9, 5'TCGAAGGCGACCTCAAGTG-3' and 5'-TTCGGTGTAGCTTT GGATCCA - $3^{\prime}$.

\section{Gelatin zymography assay}

Confluent VSMCs were maintained in serum-free medium for $12 \mathrm{~h}$ prior to being treated with GRP for $24 \mathrm{~h}$. The conditioned medium was subsequently collected, cleared by centrifugation and mixed with $2 \times$ SDS sample buffer, followed by electrophoresis on polyacrylamide gels containing $0.1 \%(\mathrm{w} / \mathrm{v})$ gelatin. After electrophoresis, the gels were incubated in renaturing buffer (2.5\% Triton X-100) with gentle agitation to remove the SDS and were then incubated in developing buffer $(50 \mathrm{mM}$ Tris- $\mathrm{HCl}$ buffer, $\mathrm{pH} 7.4$, and $10 \mathrm{mM} \mathrm{CaCl} 2$ ) overnight at $37^{\circ} \mathrm{C}$ to allow digestion of the gelatin. Finally, the gels were stained with $1 \%$ Coomassie brilliant blue R-250 and incubated in destain buffer (10\% acetic acid and 30\% methanol).

\section{RNA interference experiment}

Small interfering RNA (siRNA) duplexes for rat STAT3 and a negative control siRNA were obtained from Santa Cruz Biotechnology and Bioneer Co, respectively. Transfection of VSMCs was carried out using oligofectamine, according to the manufacturer's instructions.

\section{Statistical analysis}

Data shown are the mean \pm standard deviation (S.D.) obtained for at least three independent experiments. Statistical comparisons between groups were made by one-way analysis of variance (ANOVA) followed by Student's t-test.

\section{ACKNOWLEDGEMENTS}

This research was supported by a grant from the Basic Science Research Program through the National Research Foundation of Korea (NRF) funded by the Ministry of Science, ICT and Future Planning (2015R1A2A2A01002980).

\section{CONFLICTS OF INTEREST}

The authors have no conflicting interests.

\section{REFERENCES}

1. Majumdar ID and Weber HC (2011) Biology of mammalian bombesin-like peptides and their receptors. Curr Opin Endocrinol Diabetes Obes 18, 68-74

2. Gonzalez N, Moody TW, Igarashi H et al (2008) Bombesin-related peptides and their receptors: Recent advances in their role in physiology and disease states. Curr Opin Endocrinol Diabetes Obes 15, 58-64

3. Roesler R, Kent P, Luft T et al (2014) Gastrin-releasing peptide receptor signaling in the integration of stress and memory. Neurobiol Learn Mem 112, 44-52

4. Rao RM, Yang L, Garcia-Cardena G et al (2007) Endothelial- 
dependent mechanisms of leukocyte recruitment to the vascular wall. Circ Res 101, 234-247

5. Sprague AH and Khalil RA (2009) Inflammatory cytokines in vascular dysfunction and vascular disease. Biochem Pharmacol 78, 539-552

6. Czepielewski RS, Porto BN, Rizzo LB et al (2012) Gastrinreleasing peptide receptor (GRPR) mediates chemotaxis in neutrophils. Proc Natl Acad Sci U S A 109, 547-552

7. Kim MK, Park HJ, Kim Y et al (2017) Gastrin-releasing peptide induces monocyte adhesion to vascular endothelium by upregulating endothelial adhesion molecules. Biochem Biophys Res Commun 485, 542-549

8. Owens GK, Kumar MS and Wamhoff BR (2004) Molecular regulation of vascular smooth muscle cell differentiation in development and disease. Physiol Rev 84, 767-801

9. Abedi $\mathrm{H}$ and Zachary I (1995) Signalling mechanisms in the regulation of vascular cell migration. Cardiovasc Res $30,544-556$

10. Newby AC (2006) Matrix metalloproteinases regulate migration, proliferation, and death of vascular smooth muscle cells by degrading matrix and non-matrix substrates. Cardiovasc Res 69, 614-624

11. Sancho V, Di Florio A, Moody TW et al (2011) Bombesin receptor-mediated imaging and cytotoxicity: Review and current status. Curr Drug Deliv 8, 79-134

12. Oliveira PG, Grespan R, Pinto LG et al (2011) Protective effect of RC-3095, an antagonist of the gastrin-releasing peptide receptor, in experimental arthritis. Arthritis Rheum 63, 2956-2965

13. Ghosh A, Pechota A, Coleman D et al (2015) Cigarette smoke-induced MMP2 and MMP9 secretion from aortic vascular smooth cells is mediated via the Jak/Stat pathway. Hum Pathol 46, 284-294

14. Seki Y, Kai H, Shibata R et al (2000) Role of the JAK/STAT pathway in rat carotid artery remodeling after vascular injury. Circ Res 87, 12-18

15. Daniel JM, Dutzmann J, Bielenberg W et al (2012) Inhibition of STAT3 signaling prevents vascular smooth muscle cell proliferation and neointima formation. Basic Res Cardiol 107, 261-272

16. Doran AC, Meller N and McNamara CA (2008) Role of smooth muscle cells in the initiation and early progression of atherosclerosis. Arterioscler Thromb Vasc Biol 28, 812-819

17. Willis Al, Pierre-Paul D, Sumpio BE et al (2004) Vascular smooth muscle cell migration: Current research and clinical implications. Vasc Endovascular Surg 38, 11-23
18. Dzau VJ, Braun-Dullaeus RC and Sedding DG (2002) Vascular proliferation and atherosclerosis: New perspectives and therapeutic strategies. Nat Med 8, 1249-1256

19. $\mathrm{Yu} \mathrm{H}$, Lee $\mathrm{H}$, Herrmann A et al (2014) Revisiting STAT3 signalling in cancer: New and unexpected biological functions. Nat Rev Cancer 14, 736-746

20. Liao XH, Wang N, Zhao DW et al (2015) STAT3 protein regulates vascular smooth muscle cell phenotypic switch by interaction with myocardin. J Biol Chem 290, 1964119652

21. Dutzmann J, Daniel JM, Bauersachs J et al (2015) Emerging translational approaches to target STAT3 signalling and its impact on vascular disease. Cardiovasc Res 106, 365-374

22. Caird J, Napoli C, Taggart C et al (2006) Matrix metalloproteinases 2 and 9 in human atherosclerotic and nonatherosclerotic cerebral aneurysms. Eur J Neurol 13, 10981105

23. Wagsater D, Zhu C, Bjorkegren, J et al (2011) MMP-2 and MMP-9 are prominent matrix metalloproteinases during atherosclerosis development in the $\mathrm{Ldll}(-/-) \operatorname{Apob}(100 / 100)$ mouse. Int J Mol Med 28, 247-253

24. Mason DP, Kenagy RD, Hasenstab D et al (1999) Matrix metalloproteinase-9 overexpression enhances vascular smooth muscle cell migration and alters remodeling in the injured rat carotid artery. Circ Res 85, 1179-1185

25. Belo VA, Guimaraes D and Castro MM (2015) Matrix Metalloproteinase 2 as a Potential Mediator of Vascular Smooth Muscle Cell Migration and Chronic Vascular Remodeling in Hypertension. J Vasc Res 52, 221-231

26. Vincenti MP and Brinckerhoff CE (2007) Signal transduction and cell-type specific regulation of matrix metalloproteinase gene expression: Can MMPs be good for you? J Cell Physiol 213, 355-364

27. Sun HJ, Zhao MX, Ren XS et al (2016) Salusin-beta promotes vascular smooth muscle cell migration and intimal hyperplasia after vascular injury via ROS/NFkappaB/ MMP-9 pathway. Antioxid Redox Signal 24, 1045-1057

28. Fan Y, Mao R and Yang J (2013) NF-kappaB and STAT3 signaling pathways collaboratively link inflammation to cancer. Protein Cell 4, 176-185

29. Choi SH, Park BK, Lee KW et al (2015) Effect of respiratory syncytial virus on the growth of hepatocellular carcinoma cell-lines. BMB Rep 48, 565-570

30. Dong DD, Zhou H and Li G (2016) GPR78 promotes lung cancer cell migration and metastasis by activation of Gaq-Rho GTPase pathway. BMB Rep 49, 623-628 